\title{
Long Noncoding RNA LINC01554 as a Novel Biomarker for Diagnosis and Prognosis Prediction of Epithelial Ovarian Cancer
}

\author{
Ting Luo, Yan Jiang, and Jing Yang $(\mathbb{D}$ \\ Department of Obstetrics and Gynecology, The Renmin Hospital of Wuhan University, Wuhan, Hubei 430060, China \\ Correspondence should be addressed to Jing Yang; dryangjing@whu.edu.cn
}

Received 4 July 2021; Accepted 2 August 2021; Published 10 August 2021

Academic Editor: Fu Wang

Copyright @ 2021 Ting Luo et al. This is an open access article distributed under the Creative Commons Attribution License, which permits unrestricted use, distribution, and reproduction in any medium, provided the original work is properly cited.

\begin{abstract}
Objective. This study was aimed at exploring the diagnostic and prognostic value of long noncoding RNA LINC01554 (LINC01554) in epithelial ovarian cancer (EOC) patients. Patients and Methods. The expressions of LINC01554 in 161 EOC patients were analyzed using RT-PCR. The area under the ROC curve (AUC) was used to estimate the effectiveness of LINC01554 for prediction. The chi-square test was performed to explore the association between LINC01554 expressions and clinical characteristics in EOC patients. Kaplan-Meier assays were conducted for the examination of the influence of LINC01554 expression on the overall survival of EOC patients. Multivariate analyses were carried out to further determine prognostic values of LINC01554 expression in EOC patients. Results. LINC01554 expressions were strongly downregulated in EOC specimens compared with matched nontumor specimens $(p<0.01)$. Importantly, LINC01554 provided a high diagnostic performance for the detection of EOC specimens (AUC $=0.7827 ; p<0.001$ ). Low expression of LINC01554 was distinctly associated with the FIGO stage $(p=0.034)$ and distant metastasis $(p=0.007)$. The assays of survival data (five years) revealed that the 5 -year overall survival of the low LINC01554 expression group was distinctly shorter than that of the high LINC01554 expression group $(p=0.0017)$. Finally, in the multivariate Cox model, LINC01554 expression (RR $=2.863,95 \%$ CI: $1.185-4.421, p=0.014)$ was demonstrated to be an independent prognostic factor for overall survival of EOC patients. Conclusions. Our findings suggested that LINC01554 is an important EOC-related lncRNA, providing a potential diagnostic, prognostic biomarker and therapeutic target for EOC patients.
\end{abstract}

\section{Introduction}

Epithelial ovarian cancer (EOC) accounts for $>85 \%$ of all ovarian tumor cases, which is still a major cause of morbidity and mortality and the most lethal gynecologic neoplasm all over the world $[1,2]$. Each year, $>235,000$ new cases are estimated to be diagnosed, resulting in at least 110,000 deaths in the world [3]. Although the advances in surgery and chemotherapeutic agents have significantly improved the quality of life of EOC patients, the 5-year survival rate in EOC with advanced stages remains only $30 \%$ due to the high rate of recurrence and metastasis $[4,5]$. Thus, the molecular mechanisms underlying EOC progression need to be elucidated for the identification of novel EOC-specific biomarkers for early diagnosis of this tumor.

Long noncoding RNAs (lncRNAs), frequently dismissed as nonfunctional transcriptional "noise," are RNAs with $>200$ nucleotides in length with limited proteincoding capacity [6]. Growing studies indicate that lncRNAs contribute to different cellular processes via regulating the expression of various genes in transcriptional, posttranscriptional, or posttranslational levels [7]. Interestingly, a large number of tumor-associated lncRNAs have been characterized, and their potential roles and underlying molecular mechanisms involved in the progression and tumor metastasis have been confirmed in both in vitro and in vivo assays [6]. More importantly, several lncRNAs have been demonstrated to be detectable in serum and serve as novel sensitive prognostic and diagnostic biomarkers for various tumors, such as lncRNA DLEU1 for bladder cancer, lncRNA BRE-AS1 for prostate carcinoma, and lncRNA TC0101441 for EOC [8-10].

IncRNA LINC01554 (LINC01554), a recently identified tumor-related lncRNA, has been reported to be dysregulated 
in several types of neoplasms, such as esophageal cancer, cervical cancer, and hepatocellular carcinoma [11-13]. However, the present evidence of abnormal LINC01554 expression in above tumors was limited. For the first time, Zheng et al. [13] performed functional assays, providing robust evidence that downregulation of LINC01554 weakened the proliferation and metastasis of hepatocellular tumor cells via Akt/mTOR signaling. However, the function of LINC01554 in other tumors remained largely unclear. Here, we firstly reported the possible clinical significance of LINC01554 in EOC patients.

\section{Patients and Methods}

2.1. Patients and Tissue Samples. 161 pairs of fresh EOC specimens and their adjacent normal specimens were obtained from 161 EOC patients (age: 23-68) from the Renmin Hospital of Wuhan University with approval from the Ethics Committee of our hospital. The diagnosis of EOC was confirmed pathologically, and all samples in the histological characterization and clinicopathological staging were determined. The clinical information of all cases was recorded by using an electronic file with a long-term follow-up more than five years. Prior patients' written informed consent was obtained, which allowed us to use their clinical materials for research purposes.

2.2. RNA Extraction and $q R T-P C R$ Analyses. Using TRIzol based on the manufacturer's protocol, total RNA was extracted from all samples. A total of 600 ng RNA was converted into cDNA using the PrimeScript ${ }^{\mathrm{TM}} \mathrm{RT}$ reagent kit (Takara, Suzhou, Jiangsu, China). RT-PCR was carried out using the SYBR Green PCR Kit (Takara, Suzhou, Jiangsu, China) on the ABI 7500 Fast Real-Time PCR System (Biosystems, USA). Relative expression values were calculated by the $2^{-\Delta \Delta \mathrm{Ct}}$ methods. GAPDH was used as an internal reference. The primers used in this study were shown below: for LINC01554, CATGGAGTATCTAAGCAGCC (forward) and CAGTTGAYAGCGGAGCCTTG (reverse) and for GAPDH, CAATGACCCCTTCATTGACC (forward) and GACAAGCTTCCCGTTCTCAG (reverse).

2.3. Statistical Analysis. All data were statistically analyzed using SPSS (19.0 version; IBM, Chicago, IL, USA). Differences between groups were analyzed using Student's $t$-test or one-way ANOVA based on the types of groups. ROC assays were carried out to explore the diagnostic value of LINC01554 for EOC patients. By the use of the KaplanMeier method with the log-rank test, our group calculated the overall survival rates of all patients. Multivariate assays were carried out to test the prognostic value of LINC01554 in EOC patients. $p<0.05$ was considered statistically significant.

\section{Results}

3.1. Low Expression of LINC01554 in EOC Patients. In recent years, more and more lncRNAs were identified to be dysregulated in EOC. To explore whether LINC01554 was a functional lncRNA in EOC progression, we performed RT-PCR

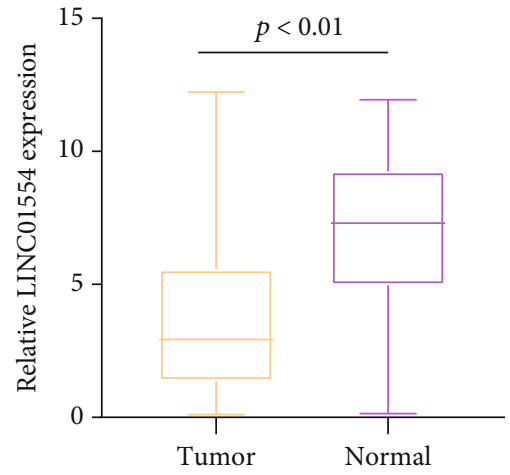

Figure 1: RT-PCR for the expression of lncRNA LINC01554 in specimens compared to matched nontumor tissues.

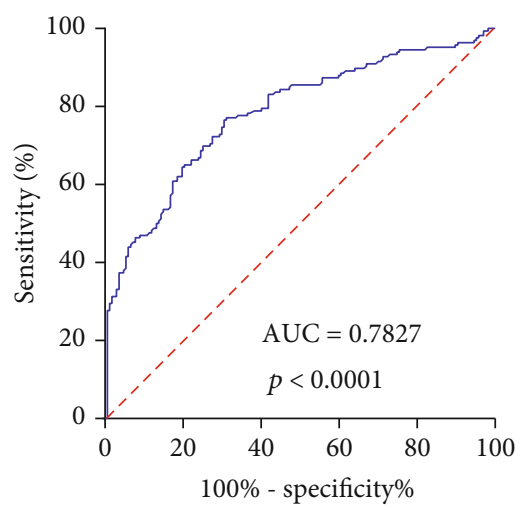

FIgURE 2: ROC curve of lncRNA LINC01554 expression to distinguish EOC specimens from matched nontumor tissues. The area under the curve (AUC) of lncRNA LINC01554 was 0.7827.

to examine its expression in 161 EOC specimens. As presented in Figure 1, LINC01554 levels in EOC specimens were distinctly lower than those in noncancerous tissues $(p<0.01)$.

3.2. The Diagnostic Value of Dysregulated LINC01554 Expression in EOC Patients. To examine whether the tissue LINC01554 had diagnostic potential, we performed ROC assays. As shown in Figure 2, LINC01554 had an AUC value of 0.7827 ( $95 \%$ CI 0.7333 to 0.8322 ) for EOC. In addition, the sensitivity and specificity of LINC01554 for distinguishing EOC specimens from nontumor tissues was $73.32 \%$ and $89.67 \%$, respectively. Overall, our findings revealed that LINC01554 may be used as a biomarker for the early screening of EOC patients.

3.3. Association between LINC01554 Expression and Clinical Features of EOC Patients. The median value of relative LINC01554 expression was 6.43 according to the test of qRT-PCR in 161 samples of EOC, which were classified into two groups (high group: $n=80$, low group: $n=81$ ). Then, the chi-square test was performed, which indicated that low LINC01554 expressions were associated with the FIGO stage $(p=0.034)$ and distant metastasis $(p=0.007$, Table 1$)$. However, LINC01554 expressions in EOC were not correlated with other parameters (all $p>0.05$ ). 
TABLE 1: Correlation between LINC01554 expression and clinicopathological features in EOC patients.

\begin{tabular}{|c|c|c|c|c|c|}
\hline \multirow{2}{*}{$\begin{array}{l}\text { Parameters } \\
\text { Age (years) }\end{array}$} & \multirow{2}{*}{$\begin{array}{c}\text { Group } \\
<55\end{array}$} & \multirow{2}{*}{$\frac{\text { Total }}{79}$} & \multicolumn{2}{|c|}{$\begin{array}{c}\text { LINC01554 } \\
\text { expression }\end{array}$} & \multirow{2}{*}{$\begin{array}{r}p \text { value } \\
0.180\end{array}$} \\
\hline & & & 35 & 44 & \\
\hline & $\geq 55$ & 82 & 45 & 37 & \\
\hline \multirow[t]{5}{*}{ Histological subtypes } & Serous & 88 & 48 & 40 & 0.721 \\
\hline & Endometrioid & 23 & 9 & 14 & \\
\hline & Mucinous & 19 & 11 & 8 & \\
\hline & Clear cell & 17 & 7 & 10 & \\
\hline & Others & 14 & 5 & 9 & \\
\hline \multirow[t]{2}{*}{ Tumor size $(\mathrm{cm})$} & $\leq 5$ & 110 & 59 & 51 & 0.141 \\
\hline & $>5$ & 51 & 21 & 30 & \\
\hline \multirow[t]{2}{*}{ FIGO stage } & $\mathrm{I}+\mathrm{II}$ & 108 & 60 & 48 & 0.034 \\
\hline & $\mathrm{III}+\mathrm{IV}$ & 53 & 20 & 33 & \\
\hline \multirow[t]{2}{*}{ Grade } & G1 & 108 & 57 & 51 & 0.263 \\
\hline & $\mathrm{G} 2+\mathrm{G} 3$ & 53 & 23 & 30 & \\
\hline \multirow[t]{2}{*}{ Distant metastasis } & Yes & 39 & 12 & 27 & 0.007 \\
\hline & No & 122 & 68 & 54 & \\
\hline
\end{tabular}

3.4. Low Expression of LINC01554 Correlates with Poor Prognoses. In the above findings, we confirmed that patients with low LINC01554 expression displayed a positive metastasis, suggesting that LINC01554 may influence the prognosis of EOC patients. We collected information on 5-year survivors from 161 EOC patients and performed KaplanMeier's assays. As presented in Figure 3, we observed that the 5-year overall survival of the low LINC01554 expression group was distinctly shorter than that of the high LINC01554 expression group $(p<0.0017)$. Moreover, in a multivariate Cox model, LINC01554 expression was confirmed to be an independent poor prognostic factor for overall survival of EOC patients $(p=0.014$, Table 2$)$.

\section{Discussion}

EOC has a high prevalence in China and ranks as one of the most relentless types of tumors disturbing the female reproductive tract [14]. Clinical reports based on several groups have suggested that the majority of EOC patients have achieved a favorable outcome due to the early diagnosis which possesses important abilities contributing to the systematic optimization of therapeutic schedules $[15,16]$. Despite the fact that several clinicopathological indexes have been applied to screen EOC patients, these factors have limited specificity and sensitivity, which require further improvements $[17,18]$. Growing studies have indicated that some lncRNAs were distinctly dysregulated in various tumors, and a large number of functional assays have confirmed lncRNAs as potential participants in cancer development via acting as tumor suppressors for oncogenes, which highlighted their potential use as novel diagnostic and prognostic biomarkers for tumor patients [19, 20].

LINC01554 was a recently identified lncRNA whose upregulation was firstly confirmed in hepatocellular carcinoma according to a previously published microarray analy-

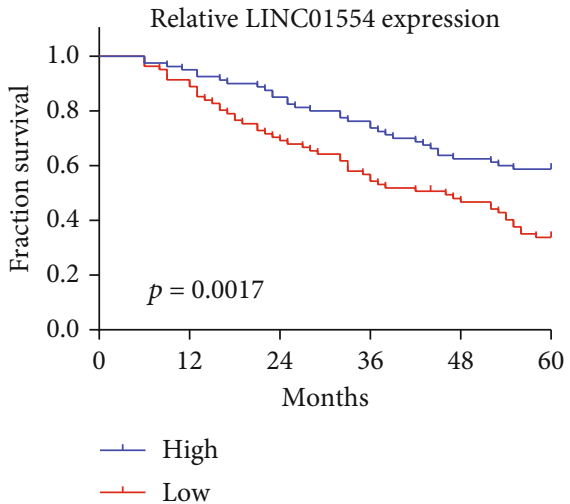

Figure 3: Kaplan-Meier survival curves for EOC patients according to lncRNA LINC01554 expression.

sis [21]. Then, a previous study by Zheng et al. [13] further provided similar evidence that LINC01554 was lowly expressed in hepatocellular carcinoma by performing RTPCR in 167 primary hepatocellular carcinoma samples. Importantly, they showed that knockdown of LINC01554 suppressed the proliferation and metastasis via suppressing the Akt/mTOR signaling pathway, suggesting LINC01554 as an oncogenic lncRNA in hepatocellular carcinoma. On the other hand, a clinical study by Ding et al. [22] confirmed that low LINC01554 expression predicted a poor prognosis of hepatocellular cancer. These findings suggested LINC01554 as a tumor promotor in hepatocellular cancer progression. However, the function of LINC01554 in other tumors has not been investigated.

In this study, for the first time, we confirmed that LINC01554 was distinctly downregulated in EOC, which was in line with its expressing trend in hepatocellular carcinoma. Then, we analyzed its clinical significance in our cohort, demonstrating that lower LINC01554 expressions 
TABLE 2: Univariate and multivariate analyses of prognostic parameters in EOC patients by Cox regression analysis.

\begin{tabular}{|c|c|c|c|c|c|c|}
\hline \multirow[t]{2}{*}{ Variable } & \multicolumn{3}{|c|}{ Univariate analysis } & \multicolumn{3}{|c|}{ Multivariate analysis } \\
\hline & $\mathrm{RR}$ & $95 \% \mathrm{CI}$ & $p$ value & $\mathrm{RR}$ & $95 \% \mathrm{CI}$ & $p$ value \\
\hline Age (years) & 1.328 & $0.667-1.832$ & 0.323 & - & - & - \\
\hline \multicolumn{7}{|l|}{$\geq 55$ vs. $<55$} \\
\hline Histologic grade & 1.441 & $0.722-1.933$ & 0.286 & - & - & - \\
\hline \multicolumn{7}{|c|}{ Serous vs. endometrioid+mucinous+clear cell+others } \\
\hline Tumor size $(\mathrm{cm})$ & 1.399 & $0.873-2.218$ & 0.185 & - & - & - \\
\hline \multicolumn{7}{|l|}{$\leq 5$ vs. $>5$} \\
\hline Tumor grade & 1.311 & $0.765-1.993$ & 0.121 & - & - & - \\
\hline \multicolumn{7}{|l|}{$\mathrm{G} 1$ vs. $\mathrm{G} 2+\mathrm{G} 3$} \\
\hline Distant metastasis & 3.219 & $1.378-5.281$ & 0.007 & 2.985 & $1.173-4.772$ & 0.013 \\
\hline \multicolumn{7}{|l|}{ Yes vs. no } \\
\hline FIGO stage & 2.985 & $1.487-4.775$ & 0.011 & 2.765 & $1.285-4.554$ & 0.016 \\
\hline \multicolumn{7}{|l|}{ I+II vs. III+IV } \\
\hline LINC01554 expression & 3.019 & $1.375-4.776$ & 0.009 & 2.863 & $1.185-4.421$ & 0.014 \\
\hline High vs. low & & & & & & \\
\hline
\end{tabular}

in EOC specimens were associated with the FIGO stage and distant metastasis. Due to the prognostic value of the positive metastasis in EOC patients, we wondered whether LINC01554 could modulate the clinical outcomes of EOC patients. Using Kaplan-Meier methods, we found that EOC patients with high levels of LINC01554 suggested a short survival time in comparison with those with low levels of LINC01554. More importantly, the results of multivariate analyses confirmed LINC01554 as an independent poor prognostic factor for overall survival of EOC patients. Although our findings provided clinical evidence on the clinical values of LINC01554 in EOC patients, the sample size is relatively small, and large clinical trials are needed to be conducted. In addition, based on our results, we suggested LINC01554 as a tumor-suppressive lncRNA in EOC. Thus, in vitro and in vivo assays with gain-of and lost-of-function experiments were needed to explore the specific function of LINC01554 in EOC cells.

\section{Conclusions}

We revealed that LINC01554 may be a novel diagnostic and prognostic biomarker for EOC patients.

\section{Data Availability}

The data used to support the findings of the present study are available from the corresponding author upon reasonable request.

\section{Conflicts of Interest}

The authors declare that they have no conflict of interests.

\section{Authors' Contributions}

Ting Luo and Yan Jiang contributed equally to this work.

\section{Acknowledgments}

This work was supported by the National Key Research and Development Program of China (No. 2018YFC1002804).

\section{References}

[1] W. Chen, R. Zheng, S. Zhang et al., "Cancer incidence and mortality in China, 2013," Cancer Letters, vol. 401, pp. 6371, 2017.

[2] M. M. Fidler, F. Bray, and I. Soerjomataram, "The global cancer burden and human development: a review," Scandinavian Journal of Public Health, vol. 46, no. 1, pp. 27-36, 2018.

[3] L. A. Torre, F. Islami, R. L. Siegel, E. M. Ward, and A. Jemal, "Global cancer in women: burden and trends," Cancer Epidemiology, Biomarkers \& Prevention, vol. 26, no. 4, pp. 444457, 2017.

[4] S. Pignata, S. C. Cecere, A. Du Bois, P. Harter, and F. Heitz, "Treatment of recurrent ovarian cancer," Annals of Oncology, vol. 28, supplement_8, pp. viii51-viii56, 2017.

[5] B. Orr and R. P. Edwards, "Diagnosis and treatment of ovarian cancer," Hematology/Oncology Clinics of North America, vol. 32, no. 6, pp. 943-964, 2018.

[6] J. Beermann, M. T. Piccoli, J. Viereck, and T. Thum, "Noncoding RNAs in development and disease: background, mechanisms, and therapeutic approaches," Physiological Reviews, vol. 96, no. 4, pp. 1297-1325, 2016.

[7] I. Blokhin, O. Khorkova, J. Hsiao, and C. Wahlestedt, "Developments in lncRNA drug discovery: where are we heading?," Expert Opin Drug Discov, vol. 13, no. 9, pp. 837-849, 2018.

[8] Z. Chen, M. Zhen, and J. Zhou, "LncRNA BRE-AS1 interacts with miR-145-5p to regulate cancer cell proliferation and apoptosis in prostate carcinoma and has early diagnostic values," Bioscience reports, vol. 39, no. 3, 2019.

[9] Y. Li, B. Shi, F. Dong, X. Zhu, B. Liu, and Y. Liu, "Long noncoding RNA DLEU1 promotes cell proliferation, invasion, and confers cisplatin resistance in bladder cancer by regulating the miR-99b/HS3ST3B1 axis," Frontiers in Genetics, vol. 10, p. 280, 2019. 
[10] J. J. Qiu, X. J. Lin, X. Y. Tang, T. T. Zheng, X. Y. Zhang, and K. Q. Hua, "Long noncoding RNA TC0101441 induces epithelial-mesenchymal transition in epithelial ovarian cancer metastasis by downregulating KiSS1," International Journal of Cancer, vol. 146, no. 9, pp. 2588-2598, 2020.

[11] Q. Fan and B. Liu, "Identification of a RNA-Seq based 8-long non-coding RNA signature predicting survival in esophageal cancer," Medical Science Monitor, vol. 22, pp. 5163-5172, 2016.

[12] X. Gang, M. Yuan, and J. Zhang, "Long non-coding RNA TMPO-AS1 promotes cervical cancer cell proliferation, migration, and invasion by regulating miR-143-3p/ZEB1 axis," Cancer Management and Research, vol. Volume 12, pp. 15871599, 2020.

[13] Y. L. Zheng, L. Li, Y. X. Jia et al., "LINC01554-mediated glucose metabolism reprogramming suppresses tumorigenicity in hepatocellular carcinoma via downregulating PKM2 expression and inhibiting Akt/mTOR signaling pathway," Theranostics, vol. 9, no. 3, pp. 796-810, 2019.

[14] X. Y. Zhang and P. Y. Zhang, "Recent perspectives of epithelial ovarian carcinoma," Oncology Letters, vol. 12, no. 5, pp. 30553058, 2016.

[15] T. V. Gorodnova, A. P. Sokolenko, E. Kuligina, I. V. Berlev, and E. N. Imyanitov, "Principles of clinical management of ovarian cancer," Chinese Clinical Oncology, vol. 7, no. 6, p. 56, 2018.

[16] P. Jessmon, T. Boulanger, W. Zhou, and P. Patwardhan, "Epidemiology and treatment patterns of epithelial ovarian cancer," Expert Review of Anticancer Therapy, vol. 17, no. 5, pp. 427-437, 2017.

[17] J. Morrison, C. Thoma, R. J. Goodall et al., "Epidermal growth factor receptor blockers for the treatment of ovarian cancer," Cochrane Database of Systematic Reviews, vol. 2018, no. 12, 2018.

[18] S. Kouba, L. Ouldamer, C. Garcia et al., "Lipid metabolism and calcium signaling in epithelial ovarian cancer," Cell Calcium, vol. 81, pp. 38-50, 2019.

[19] L. Bolha, M. Ravnik-Glavac, and D. Glavac, "Long noncoding RNAs as biomarkers in cancer," Disease Markers, vol. 2017, Article ID 7243968, 14 pages, 2017.

[20] S. Chandra Gupta and Y. Nandan Tripathi, "Potential of long non-coding RNAs in cancer patients: from biomarkers to therapeutic targets," International Journal of Cancer, vol. 140, no. 9, pp. 1955-1967, 2017.

[21] L. Chen, Y. Li, C. H. Lin et al., "Recoding RNA editing of AZIN1 predisposes to hepatocellular carcinoma," Nature Medicine, vol. 19, no. 2, pp. 209-216, 2013.

[22] Y. Ding, Z. Sun, S. Zhang et al., "Down-regulation of long noncoding RNA LINC01554 in hepatocellular cancer and its clinical significance," Journal of Cancer, vol. 11, no. 11, pp. 33693374, 2020. 